\title{
Do BL Lac Objects and FR I radiogalaxies inhabit the same galaxy environment?
}

\author{
A. Sandrinelli ${ }^{1}{ }^{\star}$ R. Falomo ${ }^{2}$, A. Treves ${ }^{1,3}$ \\ ${ }^{\star} \dagger$ \\ ${ }^{2}$ INAF - Istituto Nazionale di Astrofisica, Osservatorio Astronomico di Padova, Vicolo dell'Osservatorio 5, I-35122 Padova, Italy \\ ${ }^{3}$ INAF - Istituto Nazionale di Astrofisica, Osservatorio Astronomico di Brera, Via Emilio Bianchi 46, I-23807 Merate, Italy
}

Accepted XXX. Received YYY; in original form ZZZ

\begin{abstract}
We investigate the environments of galaxies around BL Lac objects and FR I radiogalaxies, the alleged parent populations of misaligned sources. We compare the environment of a sample of $50 \mathrm{BL}$ Lac objects at $0.1<\mathrm{z}<0.33$ with that of a sample of $90 \mathrm{FR}$ I galaxies at $0.1<\mathrm{z}<0.15$. The galaxy environment is estimated using the SDSS images in the i-band. We find that the galaxy excess density within $0.5 \mathrm{Mpc}$ around of FR I radiogalaxies is a factor $\sim 2$ larger than that around BL Lacs. This implies a reconsideration of the parent population of BL Lac objects.
\end{abstract}

Key words: galaxies: active - BL Lacertae objects: general - galaxies: clusters: general - radio continuum: galaxies.

\section{INTRODUCTION}

BL Lac objects (BLLs) are a class of sources originally characterized by significant and rapid flux variability, high polarization, and absence or weakness of spectral lines. In a seminal paper Blandford \& Rees (1978) suggested that BLLs should be explained by relativistic jets pointed close to the observer direction. The parent population was soon after proposed as Fanaroff-Riley I radiogalaxies (FRIs, Fanaroff \& Riley 1974), mainly on the basis of the extended radio emission, and the expected luminosity function assuming highly beamed sources (Urry \& Padovani 1994). The relativistic beaming was directly confirmed by the detection of superluminal velocity in the inner radio structures of some BLLs and also by the more recent observation of $\gamma$ ray emission (Madejski \& Sikora 2016). In fact the sources, with dimensions constrained by rapid variability, are transparent to photon scattering of high energy gamma rays only if Lorentz contraction is accounted for. This picture, which consented to insert quite naturally BLLs in the unified model of AGNs developed in the nineties (see e.g. Antonucci 1993; Urry \& Padovani 1995), has essentially survived for about 30 years, even if enriched in various aspects, as for instance the discovery that the weakness of spectral lines should be attributed not only to the relativistic enhancement of the continuum, but in part to be intrinsic.

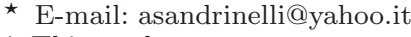

$\dagger$ This work....
}

The contribution of the Hubble Space Telescope exploration of the host galaxies has been substantial, showing that they are giant ellipticals of magnitude $\mathrm{M}_{R} \sim-22.8$, undistinguishable from unperturbed inactive ellipticals (Urry et al. 2000; Falomo et al. 2000; Sbarufatti et al. 2005).

The study of the clustering around BLLs was originally based on few objects known to belong to the class, and showed that BLLs are generally surrounded by modest galaxy groups, though some exceptions were noted (see e.g. Fried et al. 1993; Pesce et al. 1994; Wurtz et al. 1997; Falomo et al. 2014 for an overview).

The systematic survey in the $\mathrm{GeV}$ band in the last decade by the Fermi satellite demonstrated that the extragalactic sky is dominated by BLLs and greatly increased the number of known ones, which includes now more than a thousand objects (Padovani et al. 2017; Acero et al. 2015; and references therein). At the same time the Sloan Digital Sky Survey (SDSS) has covered a large fraction of the sky in various filters, so that clustering of galaxies around many BLLs can be studied in a renovated perspective with respect to some 20 years ago.

In this letter we select a sample of $\sim 50$ BLLs at $0.1<\mathrm{z}<0.33$ belonging to the SDSS DR14 and search and study the surrounding environment. We then compare with the case of $\sim 200$ inactive ellipticals from the SDSS matched in redshift and with the typical luminosity of the BL Lac host galaxies. Using the same procedure we consider also a sample of $90 \mathrm{FRIs}$, again belonging to the SDSS, in the interval $0.1<\mathrm{z}<0.15$. The results are discussed, with particular 


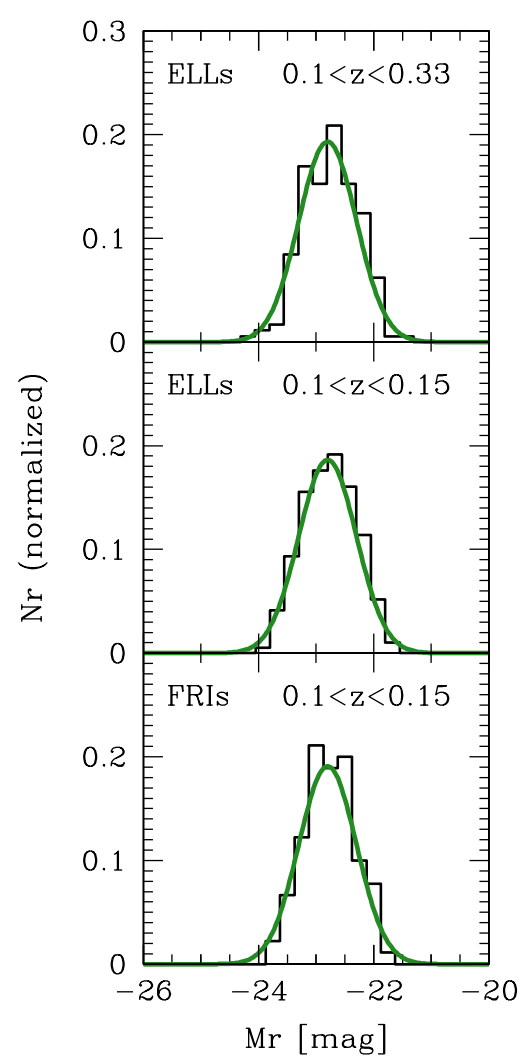

Figure 1. From top to bottom: Absolute magnitude distributions in r-band for the inactive elliptical galaxies at $0.1<\mathrm{z}<0.33$ and at $0.1<\mathrm{z}<0.15$, and for the FR I radiogalaxies at $0.1<\mathrm{z}<0.15$ in our samples. The gaussians represent the typical luminosity distribution of the BL Lac host galaxies derived from a sample of HST observations in Sbarufatti et al. (2005).

emphasis on the comparison and contrast of galaxy clustering around BLLs and FRIs.

\section{THE SAMPLES}

We searched for BLLs suitable for investigating their environment using the SDSS images and archive data. The selection of the dataset is based on the BZCAT catalog of known Blazars, Ed. 5.0 1 (Massaro et al. 2015). It contains 3,561 objects, of which 1,059 sources are spectroscopically classified as BLLs. We selected 676 BLLs with available SDSS images.

We aim to detect galaxies belonging to the environment of BLLs up to a magnitude threshold in the i-band corresponding to $\mathrm{M}_{i} \gtrsim \mathrm{M}^{*}+2.5 \quad\left(\mathrm{M}^{*}=-21.74\right.$ Montero-Dorta \& Prada 2009). To reach this goal at the completeness of $50 \%$ (Capak et al. 2007) ${ }^{2}$, we need to limit the selection to objects photometrically classified as galaxies up to $\mathrm{z} \lesssim 0.33$ (mean threshold magnitude $\mathrm{i}=21.9 \pm 0.1$ mag). This value is taken as redshift upper limit for the

1 www.asdc.asi.it/bzcat/

2 Durham University Cosmology Group, references and data in http://astro.dur.ac.uk/ nm/pubhtml/counts/counts.html

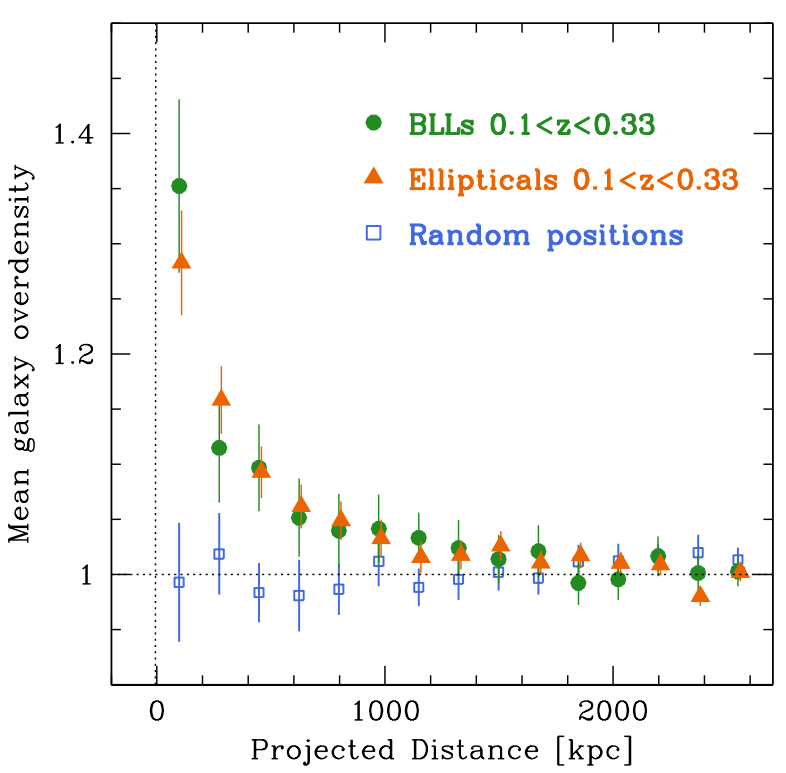

Figure 2. Average overdensity of galaxies with magnitude brighter than $\mathrm{mi}_{50 \%}$ (see text) as a function of the projected distance from BL Lac objects (green filled circles), inactive ellipticals (orange filled triangles) and random positions (blue open squares). Both BL Lacs and inactive ellipticals exhibit the same galaxy overdensity at projected distance $\lesssim 1 \mathrm{Mpc}$. The errors are the standard errors of the mean (see also Sandrinelli et al. 2018).

sources in this study. We also set a lower value to the redshift at $\mathrm{z}=0.1$ in order to limit the size of the fields. This excludes 4 targets. We consider only BLLs with reliable spectroscopic classifications and redshifts. Targets with corrupted images in the close surrounding area ${ }^{3}$ are discarded. The final sample is composed of 47 BLLs with an average redshift $\mathrm{z}_{\text {ave }}=$ 0.240 .

For a direct comparison of the BLL environment with that around inactive elliptical galaxies, we select $\sim 200$ inactive ellipticals matched in redshift with our BLL sample and in luminosity with the BLL host galaxies distribution reported in Sbarufatti et al. (2005). The targets, with spectra in the SDSS archives, are drawn on the basis of the contribution of de Vaucouleurs profile with respect to the exponential profile (p.fracdev $i>0.8$ ) from good photometry $($ CLEAN $=1)$ galaxies (type $=3$ ). For further comparisons among the environments (see Section 3), we also built a number of samples of $\sim 200$ inactive elliptical galaxies in different ranges of redshift. The luminosities of all the samples of inactive ellipticals are encompassed between $-24.0 \lesssim$ $\mathrm{M}_{r} \lesssim-21.5 \mathrm{mag}$ with mean absolute magnitude $\sim-22.8 \pm$ 0.5 (standard error of the mean $=0.03$ ), see Figure 1 and Sect. 3 .

To build a suitable sample of FR I radiogalaxies we used the dataset of Capetti et al. (2017), which contains 219 sources at $\mathrm{z}<0.15$, obtained by combining observations from the NVSS, FIRST, and SDSS surveys. Consistently with the case of inactive ellipticals we built a sample of objects at

3 We note that $\sim 30 \%$ of the objects shows an incomplete coverage or corrupted frames in the area of our investigation, which extents up to 20 arcmin from each source. 
z $>0.1$ appearing in the SDSS and matching in absolute magnitude with the BLL host galaxies, see Figure 1. Again for both the inactive ellipticals and FRI samples, targets with damaged images in the investigated areas are removed from the selections. A number of $90 \mathrm{FRI}$ galaxies are finally assembled, with absolute magnitudes $-22.76 \pm 0.45$.

\section{ANALYSIS}

We drew information from SDSS DR14 catalogues, and obtain position and i-band photometry of each target and of all primary objects classified as galaxies (type $=3$ ). Absolute magnitudes $\mathrm{M}_{i}$ of galaxies, evaluated at the redshift of the target from modelMag magnitudes, are corrected for galactic extinction based on the SDSS values and are also $K$ corrected assuming the elliptical template (Mannucci et al. 2001).

To study the galaxy environment we follow the procedure described by Sandrinelli et al. (2014; 2018). We start by computing the surface number density $n_{r}$ of galaxies brighter than a magnitude threshold (see Sect. 4), observed inside projected circular annuli of central radius $r$. The radius is evaluated at the redshift of the target and increases by steps of fixed width. The target source is excluded. We compare $n_{r}$ with the surface number galaxy density $n_{b g}$ in the outer region (from $\sim 2.5 \mathrm{Mpc}$ to $\sim 4.5 \mathrm{Mpc}$ ), taken as the background value, by adopting two parameters: the galaxy overdensity defined as $O_{r}=n_{r} / n_{b g}$, and the excess galaxy surface density $E_{r}=\left(n_{r}-n_{b g}\right)$.

\section{RESULTS}

The comparison of the distribution of galaxies around BLLs with the sample of inactive ellipticals that are matched both in redshift and galaxy luminosity shows that they inhabit the same galaxy environment (see Figure 2). The average galaxy overdensity with respect to the background is detected up to a projected distance from the target of $\sim 1 \mathrm{Mpc}$ and within $0.5 \mathrm{Mpc}$ is $O_{r} \sim 1.15$. Furthermore, we perform a sanity test by searching for overdensity applying the identical analysis around random positions, obtained with shifts of 15 arcmin from each BLL. No evidence of overdensity is found (see Figure 2) and therefore we consider robust the result of similar clustering around BLLs and ellipticals.

We now aim to compare the galaxy clustering around BLLs and FRIs. Our samples are well matched in the luminosities, but not in redshift, and the BLL sample is too small to consider only the objects at $\mathrm{z}<0.15$. Therefore we use as parameter the excess densities $E_{r}$ and first compare BLLs and ellipticals matched in luminosity and redshift. We use as threshold the apparent magnitude i, corresponding to $M_{i}^{*}+2$ at the redshift of each individual target. As illustrated in Figure 3 we find on average a very good agreement, consistently with the results on the overdensity. We have checked that this is the case also considering 5 different redshift intervals from $\mathrm{z}=0.1$ to $\mathrm{z}=0.35$, each containing $\sim 200$ ellipticals. As expected, within the uncertainties we do not find any difference between $E_{r}$ evaluated in the various redshift intervals. This is apparent in Figure 3, where the cases of ellipticals at $0.1<z<0.15$ and at $0.1<z<0.33$ are reported.

The sample of FR I radiogalaxies is limited to $0.1<\mathrm{z}<0.15$ (see Sect. 2), therefore we can only do a direct comparison with the sample of inactive ellipticals in the same redshift range. We found that within $\sim 0.5 \mathrm{Mpc}$ the excess density of the FR I radiogalaxies is a factor 2 larger than that of ellipticals (see Figure 3). Since we have shown that the galaxy environment of ellipticals does not change in the redshift range $0.1<z<0.35$, we are confident that also the comparison of environment between BLL and FR I radiogalaxies is reliable. The result is illustrated in Figure 3. In particular, within $0.5 \mathrm{Mpc}$ the excess density is $13 \mathrm{Mpc}^{-2}$ for the FRIs, while for the BLLs it is $\sim 6 \mathrm{Mpc}^{-2}$.

\section{CONCLUSIONS}

We find that the galaxy environment of low redshift BL Lac objects is indistinguishable from that of low redshift inactive ellipticals of comparable galaxy luminosity. This result is similar to that found by Karhunen et al. (2014) and Sandrinelli et al. (2018) who considered large samples of low redshift QSOs, finding an overdensity within $0.5 \mathrm{Mpc}$ of $\sim 1$. So it seems that in the local Universe BLLs, quasars and inactive ellipticals are similar in terms of surrounding galaxies.

We also find that the clustering of galaxies around radiogalaxies of FR I type is on average a factor $\sim 2$ richer than that of BL Lac objects. In spite of the difference in the redshift distribution this difference appears robust since no variation of galaxy environment is found for inactive elliptical galaxies of same luminosity up to $\mathrm{z} \sim 0.3$ (see details in Sect. 4). This result is further illustrated in Figure 4, where we report the cumulative distributions of the excess density for the two classes of objects within the projected distance $350 \mathrm{kpc}$. The medians are 5.7 and $13.6 \mathrm{Mpc}^{-2}$ for BLLs and FRIs, respectively, and the KS test yields a p-value $<4 \cdot 10^{-4}$. The population of BLLs is positioned at lower excess densities with respect FRIs.

The different clustering of galaxies around FRIs with respect to that of BLLs was suggested some 20 years ago by Wurtz et al. (1997). They considered $\sim 50$ BL Lacs at $\mathrm{z}$ $<0.65$ and a similar number of FR I radiogalaxies at $\mathrm{z} \lesssim 0.5$, without performing any matching between the two classes in terms of redshifts and host galaxies luminosity distributions.

The unambiguous distinction of FR I radiogalaxies and BLLs in terms of clustering of galaxies in the immediate environments poses a serious problem for the interpretation of the former class as the parent population of the latter. A possible explanation of this is that BLLs have as parent population only $50 \%$ of the entire class of FR I radiogalaxies, while the remaining objects with the higher clustering are not connected with the BL Lacs.

\section{ACKNOWLEDGEMENTS}

We thank Francesco Massaro for friendly and constructive conversations on the subjects presented in this letter. 


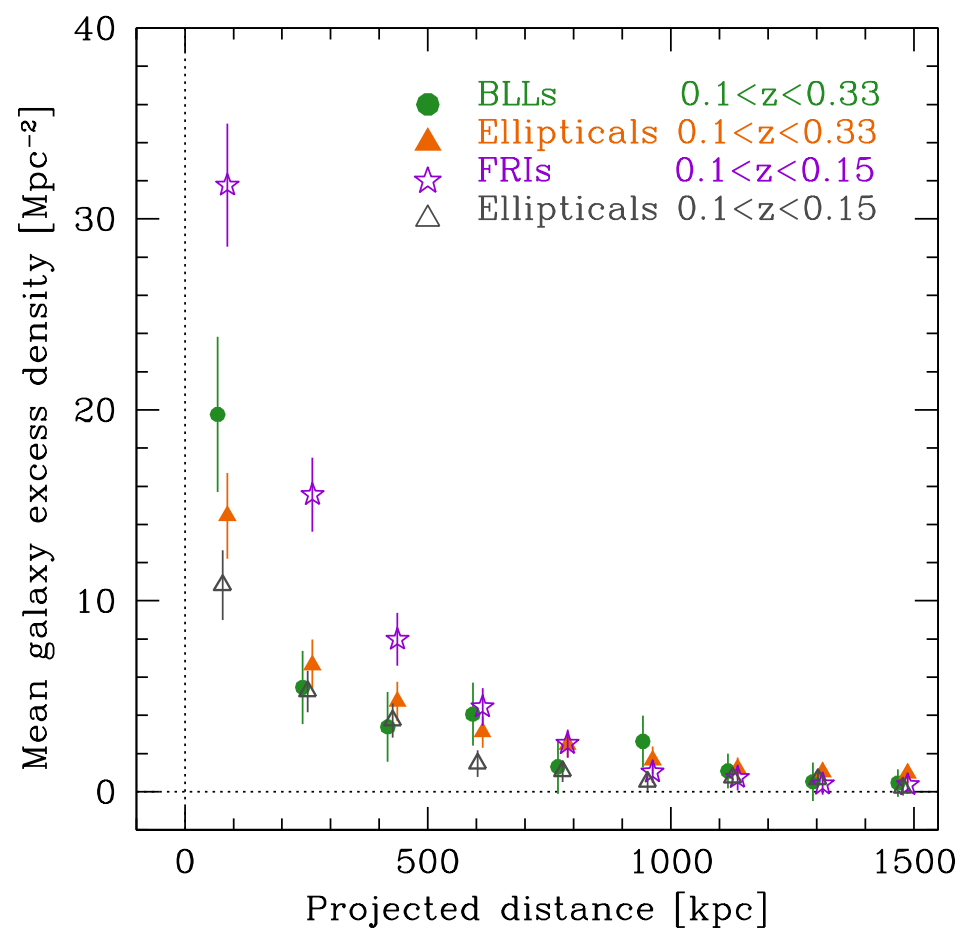

Figure 3. Average excess surface density of galaxies (detected in i-band, with magnitude threshold $\mathrm{M}^{*}+2$ ) as a function of the projected distance from the BL Lac objects (green filled circles), inactive ellipticals matched in redshift with the BLL sample (orange filled triangles), FRIs (violet open stars) and inactive ellipticals (grey open triangles) matched in redshift with the FRI sample.

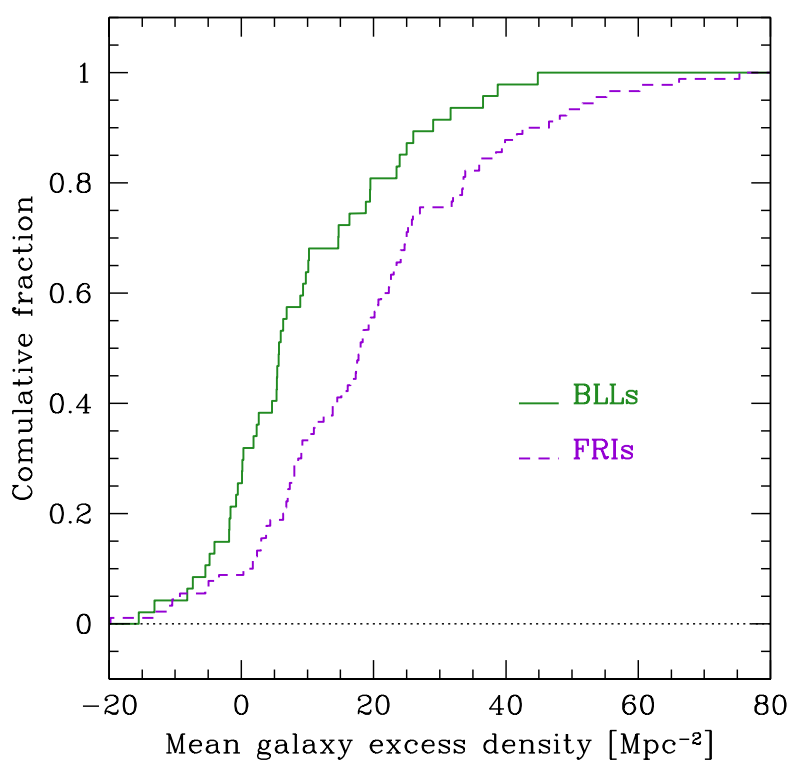

Figure 4. The comparison of the cumulative distributions of excess galaxy density (within $350 \mathrm{kpc}$ ) of BL Lac objects $(0.1<\mathrm{z}<0.33)$ and FR I radiogalaxies $(0.1<\mathrm{z}<0.15)$. The two distribution are significantly different (see text).

The use of the Sloan Digital Sky Survey facilities is gratefully acknowledged. The SDSS web site is www.sdss.org.

\section{REFERENCES}

Acero, F., Ackermann, M., Ajello, M., et al. 2015, ApJS, 218, 23 Antonucci, R. 1993, ARA\&A, 31, 473

Blandford, R. D., \& Rees, M. J. 1978, Phys. Scr., 17, 265

Capak P., et al., 2007, ApJS, 172, 99

Capetti, A., Massaro, F., \& Baldi, R. D. 2017, A\&A, 601, A81

Falomo, R., Scarpa, R., Treves, A., \& Urry, C. M. 2000, ApJ, 542, 731

Falomo, R., Pian, E., \& Treves, A. 2014, A\&ARv, 22, 73

Fanaroff, B. L., \& Riley, J. M. 1974, MNRAS, 167, 31P

Fried, J. W., Stickel, M., \& Kuehr, H. 1993, A\&A, 268, 53

Karhunen, K., Kotilainen, J. K., Falomo, R., \& Bettoni, D. 2014, MNRAS, 441, 1802

Madejski, G. , \& Sikora, M., 2016, ARA\&A, 54, 725

Mannucci F., Basile F., Poggianti B. M., Cimatti A., Daddi E., Pozzetti L., Vanzi L., 2001, MNRAS, 326, 745

Massaro, E., Maselli, A., Leto, C., et al. 2015, Ap\&SS, 357, 75

Montero-Dorta A. D., Prada F., 2009, MNRAS, 399, 1106

Padovani, P., Alexander, D. M., Assef, R. J., et al. 2017, A\&ARv, 25,2

Pesce, J. E., Urry, C. M., Falomo, R., \& Treves, A. 1994, Bulletin of the American Astronomical Society, 26, 110.11

Sandrinelli, A., Falomo, R., Treves, A., Farina, E. P., \& Uslenghi, M. 2014, MNRAS, 444, 1835

Sandrinelli, A., Falomo, R., Treves, A., Scarpa, R., \& Uslenghi, M. 2018, MNRAS, 474, 4925

Sbarufatti, B., Treves, A., \& Falomo, R. 2005, ApJ, 635, 173

Urry, C. M., \& Padovani, P. 1994, The Physics of Active Galaxies, 54,215

Urry, C. M., \& Padovani, P. 1995, PASP, 107, 803

Urry, C. M., Scarpa, R., O'Dowd, M., et al. 2000, ApJ, 532, 816

Wurtz, R., Stocke, J. T., Ellingson, E., \& Yee, H. K. C. 1997, ApJ, 480, 547 